\title{
PROBLEMAS DE LA EDICIÓN DE TEXTOS ALJAMIADO-MORISCOS
}

Cuando en el año 1609 los moriscos fueron expulsados de España, dejaron en sus casas libros manuscritos que les habían servido para mantener su fe. Los moriscos no volvieron a España y estos manuscritos, llamados aljamiados, quedaron escondidos. Más tarde, poco a poco, fueron encontrados y hoy están en bibliotecas, en España y fuera de ella. Algunos han sido publicados, pero la mayor parte está sin publicar. Ahora, quien se pone a editar textos aljamiados choca con varias dificultades. Las primeras son de orden material. El manuscrito puede tener agujeros como, por ejemplo, el 5053 de la Biblioteca Nacional de Madrid. O el papel se ha oscurecido de manera que las letras contrastan apenas con el fondo. Muchas veces los puntos encima o debajo de las letras han penetrado toda la hoja y aparecen del otro lado de manera que no se sabe a qué letra pertenecen. Así se pueden confundir $b, t, t, y, y$ y. Lo mismo vale para otros grupos de letras.

Otras dificultades resultan del sistema gráfico de los manuscritos. Rarísimos textos están escritos con el alfabeto latino. Casi siempre se nos presentan con letras árabes. Y entonces se le plantea al editor el problema de la transcripción.

Al mismo tiempo le incumbe al aljamiadista entender y explicar la lengua de los textos, es decir: debe mostrar sus particularidades lexicales, semánticas, gramaticales y estilísticas. También el contenido necesita interpretación.

Cuando el investigador se encuentra en presencia de varios manuscritos de un mismo tema, tendrá que elaborar las relaciones entre los textos. En fin, los problemas son múltiples y las soluciones no se encuentran en poco tiempo.

Desde hace poco tiempo estoy ocupado en preparar otra edición de textos aljamiados ${ }^{1}$ que tratan todos el mismo tema. Con la excepción de un manuscrito, acabo de descifrar los textos y de transcribirlos. Todo el resto del trabajo filológico queda todavía por hacer. Aún desconozco la solución de una cantidad de problemas.

${ }^{1}$ He editado ya: Aljamiadotexte. Ausgabe mit einer Einleitung und Glossar, 2 ts., Wiesbaden, 1974 . 
No puedo presentar aquí nada definitivo; tampoco la transcripción lo es. Sin embargo me parece interesante y justificado dejar participar al lector en la elaboración de una edición de textos aljamiados ya en un estadio temprano del trabajo. También sería interesante recibir sugestiones de los conocedores.

Tengo reunidos 5 textos aljamiados con el tema de la ascensión de Mahoma al cielo. Los manuscritos correspondientes son

1. Ms. J 9 de la Escuela de Estudios Árabes de Madrid (antes Biblioteca de la Junta), los folios 16-40;

2. Ms. 5053 de la Biblioteca Nacional de Madrid. Es un manuscrito de sólo 18 folios que trata únicamente nuestro tema;

3. Ms. T 17 de la colección Gayangos (en la Real Academia de la Historia de Madrid), los folios 160-180;

4. Ms. J 60 De la Escuela de Estudios Árabes de Madrid (antes Biblioteca de la Junta), los folios 133-147;

5. Ms. 1163 de la Biblioteca Nacional de París, los folios 12-60.

A partir de ahora citaré estos textos sólo por su cifra. Dos de estos textos han sido publicados recientemente, el 1163 por la señora Rosario Suárez Piñera como parte de su tesis doctoral'2; el 5053 ha sido publicado por mí como parte de los "Aljamiadotexte"’. El manuscrito 17 se encuentra en las Leyendas moriscas de Guillén Robles ${ }^{4}$. Esta edición fue más bien una obra de divulgación que no reprodujo los textos fielmente. Es necesaria una edición moderna que presente el texto de una manera exacta. Los textos 9 y 17 no han sido publicados hasta ahora. Nuestro asunto se encuentra también en el ms. 57 de la Escuela de Estudios Árabes (fols. 78-95). Pero todavía no he podido trabajar sobre él. No menciono aquí los fragmentos de textos que hay sobre el tema.

En mi exposición de problemas de la edición de textos aljamiados me concentro sobre un pasaje reducido del texto. Un trozo relativamente breve me servirá de ejemplo.

Antes de presentar esta muestra, diré algo sobre la importancia y el contenido del texto. El punto de arranque de la leyenda de la ascensión de Mahoma a los cielos es un versículo del Corán. En el primer versículo de la sura 17 leemos: "Loado sea quien hizo viajar a su siervo, por la noche, desde la Mezquita Sagrada hasta la Mezquita más remota, aquella a la que hemos bendecido su alrededor, para

${ }^{2}$ Publicación y estudio de los pasajes del ms. 1163 de la Biblioteca Nacional de París. [Tesis doctoral, Universidad de Oviedo, 1973, 2 is.] —El texto árabe, su transcripción y las notas correspondientes se encuentran en pp. 350-456 del tomo 2.

${ }^{3}$ Pp. 389-375, t. 2.

${ }^{4}$ F. GUILLÉN ROBLES, Leyendas moriscas sacadas de varios manuscritos. Madrid 1885-86, 3 ts. Nuestro texto se encuentra en el t. 2, pp. 267-298. 
hacerle ver parte de nuestras aleyas" (traducido por Juan Vernet). En vez de "nuestras aleyas" se ha traducido también "nuestros milagros". De este versículo bastante enigmático nació una de las leyendas más pintorescas, y que encontró una acogida favorable y una propagación enorme en todas las partes del mundo islámico. "Su siervo" fue considerado como Mahoma. "La Mezquita Sagrada" y "la Mezquita más remota" fueron identificadas con el templo de La Meca y con Jerusalén. De ahí subió Mahoma a los cielos. Vuelto a La Meca, contó a sus compatriotas todo lo que había visto. He dicho ya que esa leyenda llegó a todas las tierras islámicas. Un excelente ejemplo es aquella espléndida edición del "Voyage miraculeux á travers le ciel et l'enfer", publicada en los últimos años, que contiene magníficas miniaturas y que nos da el relato de la ascensión de Mahoma en lengua turca oriental y en caracteres uiguritos ${ }^{5}$. La leyenda se difundió también en la España musulmana, como nos muestran los varios manuscritos sobre el tema en lengua árabe que se encuentran en la colección Gayangos ${ }^{6}$ y como nos certifican los manuscritos aljamiados que tratan el asunto. Pero la leyenda no se detuvo en las fronteras del mundo islámico; penetró también en tierra cristiana lo que nos testimonian las traducciones latina, francesa y castellana7. Asín Palacios mostró con buenos argumentos que elementos de nuestra leyenda han entrado hasta en la Divina Commedia del Dante.

Para comprender bien nuestro pasaje debemos conocer su colocación en el texto integral. Por eso es preciso indicar sucintamente el contenido de la leyenda.

El relato empieza con el viaje del profeta de La Meca a Jerusalén sobre la famosa cabalgadura Buráq, en compañía de Gabriel. De ahí Mahoma sube al cielo. En su ascensións atraviesa las siete esferas celestiales y encuentra muchos personajes y muchas cosas sobre los cuales recibe abundante información. Entre ellos está malak almawt, el ángel de la muerte. Ahí está el árbol de la vida cuyas hojas significan la vida de un hombre. Un gallo gigantesco anuncia cada día la hora de la primera oración. Mahoma ve el paraíso y el infierno. Entre otras personas, encuentra a Adam, a Abraham, a Ismael, a Juan, a Jesús y a Moisés. Éste le prepara el encuentro con

${ }^{5}$ MARIE-R OSE SÉguY, Le voyage miraculeux du Prophete, Montrouge, 1977.

' CI. M. ASÍN PALACIOS, La escatología musulmana en la "Divina Comedia", MadridGranada, 1943, p. 452.

7 ENRICO CERULLI, $I$ " Libro della Scala" e la questione delle fonti arabo-spagnole della "Divina Commedia", Citta del Vaticano, 1949, nos da las versiones latina, francesa y española. La versión francesa fue reeditada en PETER WUNDERLI, Le livre de l'Eschiele Mahomet. Die französische Fassung einer alfonsinischen Übersetzung, Bern 1968. Una nueva edición del texto castellano se encuentra en la tesis doctoral de J. PÉREZ ALONSO, "El Miragi". Estudio introductorio y edición crítica del texto castellano del siglo XIII, Tübingen, 1980.

8 En su Escatología musulmana. La primera edición salió en 1919 y fue seguida de una polémica de más de dos decenios. 
Allah diciéndole que no debería dejar cargar a su alumına, su comunidad, con excesivos deberes religiosos. El punto culminante del viaje es el encuentro con Allah. Y aquí comienza nuestro pasaje.

Primero, Mahoma se acerca a Allah; después asistimos a un interrogatorio. En lo que sigue, Allah impone a Mahoma y a su comunidad ciertos deberes religiosos. Mahoma consigue rebajarlos a una medida aceptable. En este punto los manuscritos difieren. En unos, Mahoma se acuerda de lo que le había dicho Moisés y empieza inmediatamente a regatear. En otros, Mahoma encuentra a Moisés sólo después de haber hablado con Allah. Moisés le hace volver a Allah. Sigue un ir y venir entre Moisés y Allah hasta que Mahoma llega a la reducción deseada. Más abajo hablaré también de las intercalaciones que hay en un grupo de textos entre el interrogatorio y la imposición de los deberes religiosos. Hasta aquí nuestro pasaje. En un grupo de textos, Mahoma ve todavía el infierno antes de bajar a la tierra; en el otro grupo, Mahoma desciende sin pasar por el infierno.

Antes de continuar la discusión presento aquí las cinco versiones del texto.

El texto J 9 tiene la particularidad de que después de casi cada $a$ u $o$ final se encuentra una $h$ no vocalizada, p. ej. en dišo $h$ (f. 34 v 3 ), abi ${ }^{\gamma}$ ah (f. 34 v 4). Después de la $i$ final esta $h$ es más rara, p. ej. en konosíh (f. 34 v 8). No se encuentra después de $e$, p. ej. ke me (f. 35 r 16). Esta $h$ suplementaria es tan frecuente que no la señalo en la transcripción. Algunas veces se encuentra en el interior de una palabra, tanto en palabras españolas como en palabras árabes. Indicamos estos casos al pie de página, p. ej. ahșalah (f. 34 v 18).

Junta 9

f. $34 \mathrm{v}$ Depu" eš dišo: "Asérkate de mí, o yā* Muhammad".

5 I :-aserkéme d-él daki'a ke no abí' a ent" re mí i :-él šino

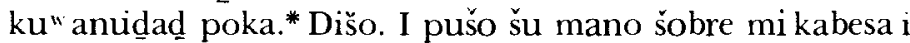
šentí šuš manoš šobre miš* pechos, porke ent ${ }^{a}$ raše el šaber en

10 mí. I konosí * toda koša en una ora. I bi a mi šeñor kon** mi korasón i non lo bi kon miš ošoš.

Depu" eš dišo a mí:

"O yā Muhammad, ¿en ké kon*ti" enden loš de la korte alta?*

Dišo a mi ${ }^{a}$ mi šeñor.

"En ganar ga radoš * altoš i v -en faser derremisi ón de

15 loš pekadoš".

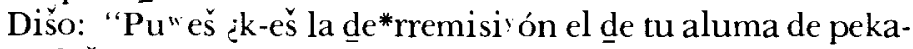
doš? *

Dišo. "La rrepentensi ‘ $a^{b /}$, e ir al-masğid, i $:-a^{*}$ tender" ell-așala" depu "eš dell-aṣala ${ }^{\text {d) }}$ ". . *
a) sigue, tachado: o yã Muhammad
d) ahșalah
b) rrebemensi' a
c) ah*tender 
20 Dišo: "Berdad diseš, o yā Muhạmmad". Dišo: "Pu" eš ¿ke šon ga rad̄os?"

f. $35 \mathrm{r}$ Diše: "Dar * a komer laš bi ‘andaš a loš pobreš kar $^{(f) *}$ ell-assalamg), i ' -el-aṣala en la noğe i laš genteš * durmi endo".

Dišo: "Berdad disě̌, o yā * Muḥammad, pu" ě̌s, o yā Muḥammad, lega * ell-așala" a tu alumma".

Depu "eš dišo a mí:

"O yā Muhammad, * yo adebdesko šobre ti i šobre tu alumma sinku"enta aṣalaeš b) ke kunp"laš * kon-eloš tú i tu

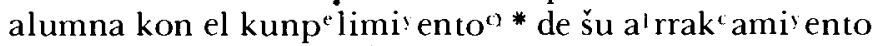
y "-asuğ"dami ento en šuš oraš, i : -ellos abrán de mí elalğanna".

10 Dišo * el mešaǧero de Alllah, faga Alllah šalutasi ón šobr-él

i šalbe: "I rremen b" róšeme * del dicho de mi ermano Músa, šobr-él šead) la šalbasi yón, * i diše: $\mathrm{O}$ mi šeñor, no podrá kon-akello * tu alumma.

15 I dišo a mi:

"O ya * Muhammad, pu"eš yo odebdesko šobre tu *alumma ku" arenta așalaešb" ke me kunpa laš * kon-elloš tú i tu alumma kon šu arrak'amis ento i '-asuğdami ento i y -el kunp limi' ento del-alwaḍ, i y -elloš abrán de mí elalğanna”.

20 Dišo el mešağero de Alllah, faga Alllah Šalutasi yón * šobr-él

f. $35 \mathrm{v} \quad \mathrm{i}$ šalbe: "I no sesé de rrogar a Alllah mi šeñor ** en-ell alinbi 'ami ento" de mi alumma dakí' a ke dišo * a mí:

"Yo adebdesko šobre ti i sobre tu * alumma sinkoașalaešbl ke" me loš * kunpalaš tú i tu alumma kon-el kunp"limi -

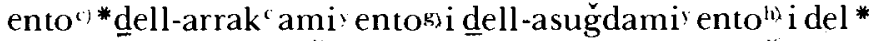

5 kunp lımi ento de šu ațahur i "-alwadữ ${ }^{i) *}$, i "-ellošabrán de mi el-alğanna.

I máš leš * daré el walardón de todoš * sinku" enta aṣalaes. bì

10 No leš me* ng "aré a elloš de šu walardón koša. I :-elloš * abrán depu" eš d-akello el-alğanna. $\mathrm{O}$ yä * Muḥammad, kiyen obrará de tu alumma * una alḥasana, eškérebill-é a él * di es alhasanaš. I ki en fisi ere un pekado* de tu alum-

15 ma, no l-ešk ribiré a él šino un pe*kado. I ši medemanda a mi perdón por el pekado, * no le l-ešk "rebiré a él pekado alguno".

Dišo: "I" -adebdesi`ó šobre mi mi šeñor šešenta di aš de ayuno.

e)bobrěs

t)bublikar

g) ahssalam

a) ahsalah

b) așalaheš

c) kunbe limil ento

e) ahlinibiyamiy entoh

f) sigue tachado: es

g) ahrak'amiventoh

d) $\bar{s}$

i) ahalwadu

h) ahsugdami: 
20 Dišo: "I " -akordóšemea) lo ke abí ' a dicho mi ermano * Mūf. $36 \mathrm{r}$ sa. í tornéme a mi šeñor i rrogéle šobre ke ** alibyaše mi še ñor a mi alumma. I kitó de mí mı šeñor * di yes di ‘aš, i man dome kon ke ayunaše sinku "enta diy aš. I tornéme a mi ermano* Müsa, i físele a šaber kon ${ }^{*}$ akello. I dišo a mí: "O ya Muhammad, no podrá * tu alumma šobr-elo-kun"pir". $\mathrm{Pu}$ " eš tórnate * a tu šeñor i rru"égalo ke alibye * Dišo: en-ell-ayuno ${ }^{\circ}$ komo te alibyó en-el-asalad".".

"I tornéme a mi šeñor, i no sesé de le rrogar, fašta * ke adebdesi' ó šobre mí i sobre mi alumma * ayuno dellayuno` del meš d-aramaḍan.

Depu "es * ke me ube umillado a mi šeñor, tanbendita * eš šu 15 noblesa, i desendí gosošo, * p $^{a}$ lasentero $\left.{ }^{*}\right)$ i hallé a Gibril kon Radiwän * el portero del-alğanna.

Ms. 5053

\section{Dešpu " eš dišso:} "Assérkate enta mí, * ya Muhammad!"

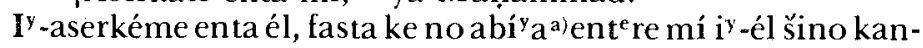
tidat muy poka, i puso su mano šobre $\mathrm{mi}^{*}$ kabesa, i ${ }^{y}$ ašentó šuš manoš šobre miš pechoš, por**ke dem "rase el šaber en mí I konosí toda koša en-una ora, i bi a mi šeñor kon qorazón i no lo bi kon miš oğoš.*

Dešpu " eš diššome: Dišse:

"Yả Muhammad ¿en ké konti enen loš * de la korte alta?

"O mi šeñor, en ganar garadaš altaš i '-en * fazer derremisi ón de loš pekadoš".

"Pu" eš ¿ke eš la de*rremisi ${ }^{y}$ ón ${ }^{*}$ de loš de alumma de loš pekadoš?"

Dišse yo:

"La rrepentensi a a ir"' a la meskida i :-ešperar la ora ${ }^{()}$delașala".

* Dišsole Allāhl:

Diššo:

"Berdad dizeš, ya Muḥammad".

$$
\text { "Pu" eš ¿̨ke * šon loš gi radoš?" }
$$

10 "Dar a komer laš bi "andaš * a loš pobreš i meskinoš ${ }^{d)}$ i publicar l-alislāme) i fazer a șsala de noche, i las genteš durmi endo".

Diššo * Allāh:

"Berdad dizeš, yā Muhammad, pu" eš pelega 1-așsala a tu alumma".
a) akordóšome
a) ' abila
e) 1-al'islàm

b) kunbe lir

c) ahyunoh

d) ahṣalah

e) bil lasemero

b) $\sqrt{\frac{3}{3}}$

c) 'ora

d) $k \sin$ vocalización 
Dešpu" eš diššome: "Yā Muhammad, yo adebdesko * a tú 15 šobre tú alumına sinku" enta a' sșalaeš en-el diva i :-en * la noche ke kunpalaz kon todo kunpi linii: ento de * šušalrrakc. aš i asağdaš en šuš oraš, i tú i " -elloš * ab"rez de mí l-alğanna." I rrekordóšele laora al al-annabi Muhammad * de lo ke abí' a f.14v dicho Músá, 'alayhi i' ssalam, i dišše "Ya mi señor, ** no podrán kunpi lir kon ello loš de mi alumına."

Dišsome: "Yā Muhammad, * pu" eš yo adebdesko śobre tú $\mathrm{i}$ šobre tu alumına $k u^{\prime \prime} a^{*}$ renta $a^{\mathrm{l}}$ șșalaeš".

I no sešé de rrogar ada ${ }^{\text {bl }}$ Allāh ke me loš * mengu "aše šobre loš de mi alumma, fašta ke diššo a mí: * "Yo adebdesko šobre tú i šobre tu alumma sinko * a'ssalaeš ke mo loš kunp" laz kon todoš šuš kunpi limi' emoš de alwadú i a țtahur i šuš a'rrak'aš i asağdaš * i tú y-elloš ab rez de mí l-alğanna. I máš leš daré el gu "alardón * de todoš loš sınku" enta a'șșalaeš, ke no leš

10 mengu" aré * ninguna koša de šu gu"alardón, yā Muhammad, ki en obrarā * de loš de tu alumma una alhasana ešk kebirl-é a él * par-ella di `ez alhạanaš. I kì en farả un pekado* no l-ešk' rebiré šino un pekado. I še me demandará * perdón por akel pekado, no le ešk "rebiré a él * ningun pekado". I dešpu "eš adebdesi" ó šobre mí i mi alumma šešenta di" aš de dayuno. I akordóšemelo de * lo ke me abíła dicho mi ermano Músā, i tornéme a mi šeñor * i rrogéle ke me mengu" aše d-elloš šobre loš de mi alumna

f. $15 \mathrm{r}$ fašta ke diššo a mí: Yo adebdesko šobre tú i šobre tu *alumma sinku "enta di" aš". I tornéme a mi ermano Músā i fízele a šaber akello i < dí >ššome:

"Yã Muhammad, no pod "rā tu alumma * kawlebarlo. ¡ $P$ Pu "eš tórnate a tu šeñor, i irruw égale en ke te ${ }^{*} m e n g u^{w}$ e en-ello komo en l-așsala!"

I tornéme a mi šeñor * i no sešé de rrogarle fašta ke adebdesi ó šobre mí * i mi alumma el dayuno del měs de ramadan.

Despu" "eš * umilyéme a mi šeñor i dile gir rasi ${ }^{y}$ aš šobre todo

10 lo ke * noš abí' a debdesido. I dešpu "eš dešpe[dí]me * de mi šñor aleg" re i pa lazentero, i fallé a Gibril kon * Riḍwān, el portero del-alğanna.

Ms.T 17

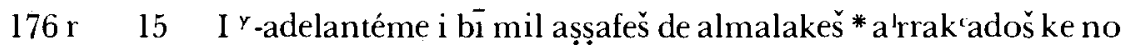
lebantaban šuš kabesaš * ni šuš pešku "esoš dikí' ’a el dí’ a del guisi`o. ${ }^{a}$

$176 \mathrm{v}$

I y -adelantéme adelante i bi beyte mil așşafeš * de almalakeš šemeğanteš de loš ot "rošb). I-škuǧe * i no oí d-elloš ningun rruido ke ya eštaban todaš laš * košaš ašošegadaš i kalladaš por mandami iento * de Allah, 'aza wa gala. 
I depu" eš' oí un pe regón ded’ Allah ke dišo: "Yā Muhammad"e) I laoraf) temh'lóronme mis ladoš * ke no loš podiy a tener ni šošegar. I oí otoro peregón * ke dizí ya: "Yā Muhammad". I

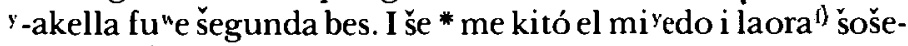
garon miš ladoš.

10 [Depu" é\} Depu ${ }^{\text {w }}$ eš oí ot" ro pergón de Alllah, tersera bes, " ke dišo: "Yā Muhammad, asérkate a mî ke yo šoy tu šeñor". I di*še yo: "Tủ eš mi šeñor, el de la o $<n>$ rragh) i de la nobleza". I dišome: "A' ssalāmu 'a lāyku, ayyuhā a'nnabī, wa

15 rahmatu wa barakātuhu". "I díšele "A'ssaíāmu almủminu al muhayminu al'azizu alğ abbāru * almutakabiru \{almutakabiru\} subḥana Alllah 'amā yušrikūna" * [Corán 59,23] I dísóme: "Yā Muhammad ¿Šabeš kon ké p" leytean * la konpaña alta?"

Dišele:" "Kon la šatišíasi $<$ yó $>$ n".

f $177 \mathrm{r}$ I díšome: ** “ ¿Ke eš la šatisfašì ón?"

I díšele ${ }^{(i)}$ "Fazer kunp ${ }^{i}$ lidamente ${ }^{*}$ l-alwaḍu a laš oraš ${ }^{i /}$

eškibaš i " -el pošarše * en la meskida ešperando l-așșala".

5 I dísome: "Yā Muhammad, * demanda por lo ke ki' eraš". Díšelea): "Señor, yo te demando fazer (loš\} loš bienešb) i ${ }^{y}$.el desendımiy ento de laš * albarakaš i y -amar a loš meskinoš $\mathrm{ku}^{\text {"w }}$ ando kerraš * kon gente de gerra. I póneme a mī y-a mi alumma bensedoreš. * I demändote, yā śeñor, tu amor i el

10 amor de amar a la obra" bu "ena* ke me aserkeš a tủ".

Dišo Alllah, noble eš en šu nob*leza: "Bì en dizeš, yā Muhamad, ¿i be" ešme, yā Muhammad? *

I dišele: "Yä mi šeñor, mi oğo me kubre la ka laredad * de tu nobleza i enfinita sesi' ${ }^{1}$ i poteštad, pero béote * kon mi korašón".

15 I díšome el noble en šu nobleza: "Yā Muhammad, gat rande eś en mi nobleza i mí fecho, $i$ alto es mi lugar. Yo šoy el poderoso de loš poderošoš * i y -el mayor de los giandes i poderošoš i l-eredero ** d-ešta a'dduni' a i de otra".

I dišo: "Berdad eš, yã mi šeñor"."

Depu "eš miré a la ešpada de gerra k-eštabe kolgada * debašo del-al'arš i ella š-ešgotiy aba šange re.

I diše yo: "O šeñor, kita de mi alumma ${ }^{\text {dl }}$ la ešpada".

5 I díšome: "Yā"Muhammad, éte enbi ' ado kon la ešpada, no še afinarä tu alumma šino * kon espada".

I dišele $e^{a i)}$ : "Yā mi šeñor, tomes a Ibrahim, * 'alayhi ilssaläm, por amigo, i fableš a Musā kon tu * palabra in-el monte de

10 Turi Sinā, i lebanteš a-Dris'is * e) en lugar alto, i perdoneš a

c) debuw es̀ akella tu" e d) da

f) la ' ora

b) sobre la línea: loš bieneš (sic!)

e) tachado (=línea 9-11): de loš poderošoš y-el mayor de loš gardeš i el-e*redero d-ešta a ${ }^{1}$ dduniy a i de la : otra. Diše: Berdad dizeš, * ya mi šeñor. Depu " eš miré a la ešpada de gerra keštaba 
12 Dāgūd pekado garande, i dište * a Sulaymān rreišmo ga rande.

15 ¿Pu" eš ké eš akello * ke le darāš a tu a'nnabí ešta noche tan ga rande i tan enša*lsada?". Díšomne el noble en šu nobleza: "Yä Muhammad, še yo tomé * a Ibrahïm por amigo, é tomado a tú, yā Muhammad, por amado. * I l-amado eš $178 \mathrm{r}$ mayor gado ke l-amigo por šetenta ** rredobleš. I še yo fablé šobre el monte de Ṭuri Sinā * kon mi palabra a Musā, yã Muhammad, éte fablado šobre loš ši ete * siy eloš. I ši puyé a Idris en lugar alto al siyelo * ku warto, a tú, yã Muhammad, éte puyado al-al' arš i ${ }^{y}$-en lugar * ke nunka lo bi ${ }^{y}$ ó almalk

5 aserkado ni a' nnabi enbiy ado. * I ši yo perdoné a Da'ūd pekado ga rande, a tú, yā Muḥammad, * éte perdonado el pekado ke aš fecho i por fazer, i t-é *dado el rri ${ }^{y}$ o del Kawsar de Alḥayawān.I ši yo di a Sulāymān* rreišmo ga rande, a tú,

10 yã Muhammad, éteit ' dado "Alhamdu lillahi" * i l-asura de albakaratu i y -el meš de Ramažan. Sepaš * ke ki y en lo šigirá de tu alumma i lo dayunarā, šerl-ā ešk ${ }^{i}$ rito * al en-el medi ${ }^{y}$ o pirimero el perdón is s.en-el medi `o ${ }^{*}$ sagero el-apagami yento de mi nobleza".

I díšele laorab): "Soy kontento, * yã šeñor".

15 Dep"éš penšé de desender de šu poder*i y-el noble i bendicho i muy onrrado $\mathrm{k}^{\mathrm{e}}$ resiy éndome * en-onrra, i díšome: "Yã Muhammad, yo ky ero adebdeser šobre tú * debdoš."

$178 \mathrm{v} \quad$ I diše yo: "El-oir" i '-el-obedeser eš a tú, $\{$ yả $\}$ ** yã šeñor".

I ${ }^{y}$-adebdeši ${ }^{y}$.ó šobre mí mī šeñor * \{ \{̌obre mī mī šeñor \} šinku" enta a'șșalaeš en-el diy $a^{*} i$ end) la noğe.

Depu" eš deballé de šu poder en la mešma manera ke*puyé, daki' 'a ke llegé a Sidratu Elmuntahā. I fallé a Gibri'il, * 'alāyahi 5 i'ssalām, en šu lugar. l a šu mano derecha abí a un * choben. I diše yo a Gibri'il: "Kiy én eš ěste ğoben * k-eštá a tu koštado?" Díšome: "Yā Muhammad, éšte eš * tu ermano Musā, 'alayhi i'ssalām, Adelántate, yā Muhammad, i da * a'ssalām šobr-él

10 I ' -adelantéme i di a'ssalam šobr-él. I tornó * l-assalām šobre mí i díšome: "Yã Muhammad, bi en ș̌eaš * benido. ¿Kómo eš tu benimiy ento de tu šeñor?"

Dísele: * "Yã ermano Musā, mi šeñor a abido $p^{a}$ laze $<>$ kon m̄̄i $i$ me a a*serkado a él aserkamiy ento muy g "rande i $\%$-a ade*bdesido šobre mî sinku" enta a așşalaes en-el di a * ${ }^{y}{ }^{y}$-en la 15 noğe".

I díšome: "Yā Muḥammad, tu alumma šerá $\mathrm{f}^{\mathrm{a}}$ laka, * no

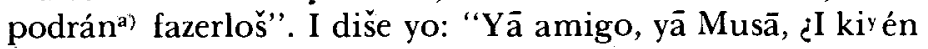

c) oy ir

d) $!$

a) bodran 
ta rašpašará laš marabillaš ke yo é bišto ** šino Allah, mi šeñor?"

I ${ }^{y}$-asi komo yo falaba ${ }^{\mathrm{b})}$ a esto, * oí un pergón de parte de Alllah, onrrado es i noble: "Yã * Muhammad, rru" ega ke yo soy šerka, obdesiy ente". *

5 Dišo el-annabì, "alayhi i'ssaíām: "I lebanté mi kabesa * a Sidratu Elmuntahā, i bíla ke ya še rrekolgaban * šuš ramoš de munğa $k^{a}$ laredad. I díšome mī šeñor: "Ya t-é amenwado dives d-elloš". I torne a mī ermano Musā. I díšome: "Yā Muhammad, torrna a

10 tu šeñor i demāndale alibyanesimi "ento d-elloš". I no sešé * de torrnar a mi šeñor, i Musā fiziy éndome fazer * dikíy a ke me loś tornó a sinko $\mathrm{i}^{1}$ ș̣alaes ${ }^{(0)}$ por su ga rasiy a.

I díšome Musā: "Tórnate a tu šeñor *i i demándale alibyanesimiy ento d-elloš ke tu alumman šerá $\mathrm{f}^{\mathrm{a}}$ laka ke no podrán fazerlos"”.

$179 v$

15 I díšele: "Yã mi ermano Musā, ya é bergu "ensa de mỉ šeñor de demandarle ** menos̉ de akešto".

I díšome: "Yā Muhammad, ya t-é dado "a tú y-a tu alumma el gu "alardón de loš šinku "enta * en-éštoš sinko i faré pešadoš šuš * pěsos̆ $d$

5 kon-elloš i daré pašada de šuš pe*kadoš".

I dišo l-annabī, 'alayhi i'ssalám: "I tomóme de * la mano Ğibi ril'i ' alayhi iıssalām, i púšome en l-alchanna i demošforóme el rríy o de Salsa *bīl i dísole: "¿Para kiy en eš ešto, ya Gibiri'il?" " Díšome: "Yā Muhammad, ešto eš para tú i para tu alumma".

10 Dišo l-annabī, șallā Allahu " alayhi wa sallam: "Depuw eš * desendimos de siy elo en si y elo fašta * ke llegamos a la eškalera. ..."

JUNTA 60

f.144v

Depués fuemos más adelante ** i bid mila) açafes d-alma*laques açachedados que no lebantarán* ${ }^{*}$ sus qabeças ${ }^{\text {b) }}$ fasta el día del juiçio'?

5 Dixo el a*nabí, çala Ałłlachu alaychi gua çalan": y a*delantéme \{y adelantéme delante y beos con ${ }^{\mathbf{1}}$ * cienti mil almalaques adalafes senblan *te ad aquellos. $Y$ començe' a-squchar y no bi * ruido ninguno. $Y$ beos con ${ }^{d)}$ todas las ${ }^{\text {(j) }}$ cosas i que *eran

10 sosegadas. Y beos con un pergueno de * partes de Alkła, aza gua jala" "), que decía: "Ye *Muchamad". Y laora tremolaron mis la*dos y no se sosegaron unos con ${ }^{\text {d) }}$ otros.

$Y$ be*os con d) un pergueno de parte de Ałła, aza * gua jalab),

15 terçera begada que deçía: "Açér"qate a mí que yo soy tu señor." y Dixe yo: "Tú eres * mi señor". Y dixei): "Atachietu
b) flaba
c) "l" $\mathrm{l}$ "s" sin vocalización
a) 3 letras aprox. ilegibles; después tachado: mala
d) entre "pe" y "šos" 2 letras tachadas
d) gon
e) somençé
t) todos los
b) qabeca
c)guiçio
1. sallā Llähu calayhi wa-sallam 2. cazza wa-ğalla
$\begin{array}{llll}\text { g) çosas } & \text { h) gala } \quad \text { i) tlxe }\end{array}$ 
lilachi, azaqui *etu li lachi, ataibetu, açalaguetu li lachi $3 \%$ " * Y dixo a mí: "Açalemu alaycqa ayuche * anabiu, gua rahmatu Ałłłachi gua baraque "tuchut". Y dixe yo a él: "Alçalemu almuminu " almuchayminu alazizu aljaberu ${ }^{\mathrm{j})} \mathrm{al}^{*}$ mutaqabiru çubhana Ałtłachu ame iuj* riquna ${ }^{k, 5\}}$ '.

Y Dixo a mí:

"Ye Muchamad, ¿̇sabes en qué * pleitean la qonpaña alta?" Y dixe" a él:

f. $145 \mathrm{r}$ "Con") la sastifaçión".

Y dixo a mí:

"Y qué eš 'm??"

Dixe"l) a él:

"Facer conplidamente") el alguador en las oras esquibadas,

y el ir a las meçuitas en las * mañanas con" las alchamas, y el sentarse * en las meçquidas a-sperar la ora del açala".

f. 145 r 6 Y dixo a mí: "Ye Muchamad, demanda por lo q*ue quieras". $Y$ dixe: "Señor, y a te demandoa) fa"zer los bienes, y el debałłłamiento de las albara*qas, y el llamar a los meçquinos ${ }^{\text {b). }}$ Y

10 quando que* ras $^{\text {' }}$ con ") las gentes gerar, bénganos de los me*nos de la gera. Y antes te demando, ye señor, $t u^{*}$ amor, $y$ el amor de tu obra, y el amor de qui*en te ama, y amor de obra que me acerque a tú".

Y dixo: "Y diçes berdad, ye Muchamad, ¿besme, " ye Muchamad?"

Dixe: "No mi señor, que mis ojos") me qubren la claredad de tu esençia y de tu nobleça. Enpero béote con "d) mi coracón'l)". Dixo * a mí: "Ye Muchamad, grande es mi fecho, y alto es *

20 mi lugar, y soi el poderoso de los poderosos, y el maior de los grandes, y el eredero del adunia y de * la otra bida."

Y dixo el anabí, çala Ałłłachu a "laichi gua çalan: "Berdad es, ye mi señor". "

f. $145 \mathrm{v}$ Después miré a la espada de la gera qu-era col*gada de suso del-alargi, y ella se goteaba de san"gre. Y dixo a él: "Ye mi señor, tira de mi aluma la-spada de la gera".

5 Dixo a mí: Ye tu aluma sino condl * espada".

Y dixo: "Ye mi señor, tomes a Ibra"chim por amigo, y fables a Muçe cond' la pa*labra, y lebantes a Idriç en alto lugar, **y perdones a Deud") pecado ${ }^{(1)}$ grande. Y distei)* a Çulaimen el

10 reismo grande ¿Y qu-es aque*llo que darás a tu anabí el día de oy, ye señor?"

Dixo a mí: "Ye Muchamad, si yo tomé a Ib"rachin por amigo, a tí é tomado por ama*do, ye Muchamad. Ell-amado es más gran

$\begin{array}{lllll}\text { j) algaberu } & \text { k) jugriquna } & \text { l) dixo } & m \text { ) os } & n \text { )dixo }\end{array}$

3. at-tahīyātu li-llāhi, az-zākiyātu li-llāhi, aț-tayyibātu, aṣ-şalawātu li-llāhi.

4. as-salāmu c alayka yā an-nabīyu wa-rahmatu Llāhi wa-barakātuhu

5. as-salāmu l-mu'minu l-muhayminu l-ç̉abbaru l-mutakabbiru subhāna Llähi canma yußrikūna. Sura 59, 23.

a) sigue tachado: do

b) mecquinos

c) $\bar{q}^{*}$ ras

d) çon

e) ogos

f) soraçon

g) Deud

h) peçado 
f. 145 v 13 ban * taja ${ }^{\text {a) }}$ qu-el-amigo ${ }^{b)}$ por setenta redobles. Y si $*$ fa15 blé con') Muce fablamiento, pues a tí * fablé sobre los siete çielos. Y si puié a Idriz * a lugar al to, al çielo \{cielo\} quarto, a tí ye * Muchamad, éte puiado a lugar que nunca"l lo bi*bió

20 almalaque çerqano ni anabí enbia*do. Y si yo perdonéc a Deud pecado" grande, a ti, * ye Muchamad, ya t-é perdonado en peqado el * $\mathrm{q}$-as fecho y es por façer. Y t-é dado el río

f. $146 \mathrm{r}$ d-Alqauçar * y el río Çalçabil. Y si di [y si di] a Çulaimen re*ysmo grande, a tí é dado, ye Muchamad, alcham*du li lachi, rabí ilalamina" y la çora de ali"f le min deliqa ${ }^{21}$, y el 5 mes de ramadan onrado sobre * que tu alma, ye Muchamad, quie<n $>$ lo acosigirá(b), * será escrito ${ }^{\text {b) }}$ en la meitad primera el perdón, y en * la meitad çagera el pagamiento". Y Dixo * a mí: "Ye Muchamad, ¿eres contento?" Dixo: "Sí, " ye mi señor".

10 Dixo el anabí, çala Ałłłachu alai*chi y çalen"'. Después pensé deballar de su poderío * y él, aza gua jalai)t), faría açala sobre mí: "Ye Mucha"ma, yo quiero adeudeçer sobre tí deudos". Y dixo: " "Ye señoril, el uir y el obadeçerk"'. Y adeudeció sobre *

15 mí y sobre mi aluma çincuentall açalaes, y e*n el día i la noche. Dixo l-anabí, çala Ałłłachu * alaichi gua çalan ${ }^{3)}$ : Debałłłém' de su poderío da *quia que lllegén' a Çidrati Ylmuntahe y tro"bé allí a Gibril, alaichi i çalen "s)", parado en *

20 su lugar, y a su mano derecha un mançebo * y dixe: "Ye Gibril, ¿y quien es aquesti mançebo * qu-está en par de tí?"

f. 146 v Dixo a mí: "Ye Muchamad, * aquesti es tu ermano Muce, alaichi i çaleny), "* adelántate i da açalen sobr-él".

$146 \mathrm{v}$ I Y adelan*téme y di açalen sobr-él. Y tornó el açalen * sobre mí y dixo a mí: "Bien seas benido, ye * Muchamad, ¿Cómoa)

5 es tu benida de tu señor?" * Y dixe a él: "Ye mi ermano, ye Muçé, que mi señor a ubido plaçer con "' mí y me a çer"qadoa él y a deudeçido sobre mi, cincuenta ${ }^{()}$* acalaes en el día y en

10 la noche". Y dixo "a mí: "Ye Muchamad,qata que tu aluma * será flaca ", que no podrán bastar") a cincu*enta"'acalaes". Dixe a él: "Ye mi hermano, ye * Muce, ¿Y quién podría pasar las marabillas "qu-é bisto de mi señor?"

Y como' yo l-é fablado, * beos con ${ }^{\text {b) }}$ un pergueno de partes de

15 Altła, aza gua jala", que deçia: "Ye Mucha*mad, ruégame que yo soi çerqa que sigo * la rogaria de quien me ruega". Dixo: "Y lle"banté mi cabeça" a Cidarati Ylmuntahe * y bila
a) bantaga
g) açosigirá
b) agio
h) esçrito
c) çon
i) gala
m) debattla
1) al-hamdu li-llāhi rabbi l-r-alamina.
2) Sura 2.
3) șallā Liāhu c alayhi wa-sallam.

d) nuça

j) senor

n) Illega

e)perdome

k) obadeçer

f) peçado
a) çomo
b) con
c) sinçuenta
d) flaça
e) bostar
I)gala
g) çabeça 
20 que ja s-escolgaba $\left\langle n>^{\text {h' }}\right.$ sus ramos de la * mucha claredad $Y$ dixo a mí mi señoril: * "Ya t-end-é quitado d-ellos dieç".

f. 147 r Y torné a mi *ermano Muçe y díxome a mí: "Torna a tu ** señor y demándale alibianeçimiento * d-ellos". Y torné a mi señor, y Muçe me açia * bolber daquía que los tornó mi señor * çincoil açalaesk' por su graçia. Dixo a mi mi * ermano Muçe:

5 "Tórnate a tu señor $\mathrm{i}$ dem"ándale alibianeçimiento que tu alu"ma es flacad/ que no podrán cunplirl' so"br-ellos". Dixo él: "Ye mi ermano Muçe, ya * é beguença de demandarle a mi seño $<r>m e$ "nos daquestos". Dixo a mí mi señor: "Ye

$10 \mathrm{Mu}$ *chamad, ya t-é dado a tú el gualardón y a * tu aluma de los cinquenta açalaes en * cincoîl açalaes, y faré pesados sus pesos, " y daré pasada se sus pecados"it)".

15 Dixo el a*nabí Muchamad, çala Ałłachu * alaichi * gua calam:

"I tomóme de la mano $\mathrm{Gi}$ *bril, alaichi içalen, y metióme en el. al"jana'al y paróme sobre el río Alqauçar * y el río de Çalçabil". Y dixe: "¿Para qui*én son estos dos ríos, ye Gibril?" Díxome a " mí: "Ye Muchamad, aquestos son pa"ra tú

f. $147 \mathrm{v}$ i tu aluma". Dixo el anabí, çala Ałła ** alaichi gua çalan: "Y denpues deballa*mos por la escalerab) ...

Ms $1163^{*}$

f. $48 \mathrm{r}$

Depu" es fu" emos a! de*de/lante.

Veos kon mil așāfes/de almalakes' ar.rak'adas, ke no se// levantaban, i -y ellos a' taçbihaban ada/Al.lah, tan bendicho es, tan alto./Depu"es fu" emos ${ }^{2}$ adelante. Veos/a nos kon

5 mil așāfes de almala*/kes açajdados, ke no alẹaban/ sus kabeças d-ik-' a el dí[' a]/del judiçi' o, i-' ellos a'taçbiḥaban/ /ada Al.lah, tan bendicho es, i tal alto./

10 Depu" es miré adelante de mi, i -v e*/skuché kon mi oreja,

f. $48 \mathrm{v}$ i no oí, sentí./Veo kontadas las kosas//ke se ibí $a^{3}{ }^{3}$ dado de mano, i s-ebi' an/rreposado por la nobleza de Al.lah, tacalā.

Veos/kon un giritán de par de Al.lah, ta'alā:/

"Pu"elvite, (sic) yā Muhamad."

5 La ora t'remolaron*/mis lados, i -y' espartínse/mis konjunturas.

a) algana

b) esçalera

- La Señora Suárez ha sido tan amable de permitirme la reproducción de su texto. Aprovecho la ocasión para agrecerle cordialmente. Reproduzco ese pasaje con la grafía que le ha dado la Señora Suárez.

$\begin{array}{llllll}\text { h) esçolgaba } & \text { i) senor } & \text { j) çinço } & \text { k) acalaes } & \text { 1) çunplir } & \text { m) peçados }\end{array}$

1 Hay un tašdid superfluo sobre el mim: almmalakes.

2 *Fur emos; "fu" e mas. Sobre el mim hay dos vocales: fatha y damma.

${ }^{3}$ El alif inicial lleva dos vocales, fatha y damma: "ubiy an: "ibiy an. 
Peos[sic] kon - un/giritante, de par de Al.lah, ta'alã, se/gunda vegada ke dezí ${ }^{y}$ :

-Yã Muḥammad,/no ayas miy edo, rrapósate anta ${ }^{4}$

10 akello.*/ Veos kon-un giritante de par de/Al.lah, terçero, ke dezíl a:

f. $49 \mathrm{r} \quad-$ Açérkate//a mí, yã Muhammad, ke yo so kon tú, ke yo/soy tu Señor.

I kedé turbado, ke/no sabi ${ }^{y}$ a kómo dase a'ççalām a mi Señor,

5 e puso mi Señor en ke diši ${ }^{y} e^{*} e^{*} /$ " $^{\prime}$ tahiyätu li-l.lahi a'zzäkiyãtu li-l.lahi /ay attayyibātu a'ș̣alawatu li-l.lahi", "las rre/verenciyas son \{verençi ${ }^{y}$ as/ son $\}$ ada Al.lah, los linbi ${ }^{y}$ ezas (sic) son/ada Al.lah".

Dišo a mí mi Señor:

0 - A'ççalảmu* / calayka ayyuhả a'nnabiya / wa-rahmatu Al.lahi wa -barakātuhu.//

f $49 \mathrm{v} \quad K^{2} k^{y}$ ere dezir: la salvac'ón sea/sobre tú, ol alnnabi, ími pi yadad, i mi ben/deci`ón. ${ }^{5}$ I dišo:

${ }_{-} \mathrm{O}$ ! mi Señor, el -aççalām/noble, onrrado, el ga randdísimo,

$5 \tan ^{*} / \mathrm{g}^{\circ}$ lorefikado es, no ả Señor $\mathrm{fu}^{\mathrm{w}} \mathrm{ara}^{6} \mathrm{de}$ tú, $/ \mathrm{ni}$ Señor a otro sino a tú. Dišo: Dišo:

-Yã Muhammad,/ ¿sabes en ké pe leyteen la konpaña?

- Por la derremiçi ón.

Dišo:

-I ké/es?, ya Muahammad?

Dišo:

f. 50r ñanadas ir a las meçkidas, $/ / \mathrm{i}$ sentarse en -ellas kon el $\mathrm{rre} / \mathrm{swardami}$ ento de las a' șalaes./Dišo:

-Dizes verdad, yã Muhammad, pu"es/demanda de mí lo que kerrás. Díšele:*/

5 -Señor; yo te demando fazer los bi ’enes, $/ \mathrm{i}$ dešar las eskivevezas, i -yamar/a los pobres, i demāndote, Señor,/tuamoríyo, i -" ell -amorí" o de toda obra ke se/acerca a tú. Diše:

10 -Dizis verdad,*/yả Muḥammad, ¿véesme kon tus ojos?/ Diše a él:

f. 50v - No, yā mi Señor, enbero,//véote kon mi çoracón. Dišo:

-Ya Muhammad,/garan es mi afer i -salto es/mi lugar,

5 el noble de la nobleza, i mante/nedor del - adduni' a, i. -y $\mathrm{el}^{*} /$ eredero de $\mathrm{ki}^{y}$ en es sobr-ella./Diso (debe ser: diše)

\footnotetext{
4 Se lee anta, por enta o ante. Al faltar el alif de prolongación no se puede decidir de qué forma se trata.

${ }^{5}$ Bendiçi ${ }^{y}$ ón. El dal lleva dos vocales, fatha y kasra. Puede deberse a una vacilación del vocalismo o a simple descuido.

${ }^{6}$ Falta el alif de prolongación tras el waw para que diga: fuera, pues un cambio vocálico de ué >uá es improbable.
} 
-Yā mi Señor, verdad es/akeso.

Dišo depu "es:

f. $5 \mathrm{lr} \quad 10$ la vengança rreskolgada en*/1 -a ${ }^{\prime}$ ars ke goteaba sang"re. $/ / \mathrm{I}$ diše:

—Yä mi Señor, alça la e/spada sobre mi aluma.

Dišo:

-Eviy arté$^{7} /$ kon la espada, no eskandalizes/tu alumma, sino kon la espada. I disě:*/

-Yā mi Señor, tú tomas ${ }^{8}$ a Ibrāhim/por amigo, i hablas a Mūçā hablamiy en/to, i perdonas a Dāwud su pekado muy $\mathrm{g}^{\mathrm{a}}$ randísimo, i donas al Culäymān $\mathrm{rreismo} / \mathrm{g}^{\mathrm{i}}$ rande, $\mathrm{pu}^{\mathrm{w}} \mathrm{es}$, ¿ké es akello ke as/dado oy a tu a ${ }^{1}$ nnabī Muhammad? Dišo Al.lah:*/

f. 5lv $10-Y a ̄$ Muhammad, si fu" é ke tomé a Ibrāhim// por amigo, $\mathrm{pu}^{\mathrm{w}}$ es ya é tomado a tú por amado, $/ \mathrm{i}$-Y el amado es más apantajado (sie) $\mathrm{k}$-el amigo/kon setentamil rradobla-

5 mi ${ }^{y}$ entos,/i si fu" e ke hablé a Müçā fablamiy ento," $/ \mathrm{pu}^{\text {wes }}$ yo te hablé sobre el monte/de Țüriçinā, a tí, yā amado, é hablado inçima/de los si ${ }^{y}$ ete çivelos. i si fu" $/$ ke alçé a Idriç

10 en lugar alto, pu" es/yo lo subí al çielo $\mathrm{ku}^{\mathrm{w}}$ arto, a tí e

f. 52r alçado*/a lugar alto, ke no te verá almalak//çerkano, ni $a^{\prime}$ nnabi envi' ado, i si fu" / ke perdoné a Dāwud, pu"es ya te

5 é dado a ti,/yā Muhammad e[1] rríy o de Alkawzar, y ${ }^{-y}$ el rriy o/de Çalçabil, i si di a Çulayman, fijo*/de Dāwud rreis-

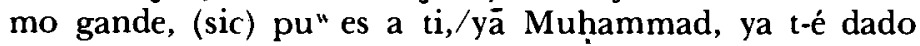
1-açūra de "alḩamdu li-l.lahi", i l-açūra de ALM dēlika", i y el me[s] de Ramadän,/pu" es sepas, yā Muhammad, ke kiven/

f. 52v lo alkançará de tu alumma, i -l dayunará, perdonaré//a él sus pekados; ¿akontén/taste kon akello, yā Muḥammad? Dišo:/

-Yo konténtome, yā mi Señor.

5 Depu" es/pensé kon deçender del poder*/de mi Señor, i díšome mi Señor:

-Yā Muhammad, demanda/de mí lo ke kerrás, dišo.

Yã:mi señor, i mi Mayor, perdóname a mí y -mi/alumma. Dišo Al.lah, tac alā:

10 Yã é fecho/akello kon stentamil de tu aluma*/kjy en se adebdeçe de fu" ego./I diše:

-Keréçeme, yã mi Señor, i mi Mayor.//

f. 53r Dišo Al.lah, tar alä: I dišo:/

-Ya é fecho graçi $^{y}$ a/a ellos, a kada uno setentamil.

7 El pasaje que precede es difícil de comprender. F. Guillén Robles Ley. Mor. T. II, p. 295 pone ${ }^{\text {¡Oh }}$ mi Señor! quita de mi pueblo la espada. — iOh! ¡Mahoma! hete enviado con la espada: no se afinará tu pueblo sino con espada".

8 Tomas, está escrito de tal modo que el ta y el mim tienen unos rasgos hacia arriba que semejąn alif de prolongación y que no son más que hábito del copista, que se repite en la línea

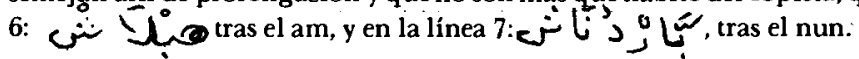


-Yā mi Señor, k'réçeme a mí.

5 I donóme/t' res rretençiy ones ke no*/konpe renderíy ${ }^{\text {y }}$ an su $\mathrm{ku}^{\mathrm{w}}$ ento/sino Al.lah. I diše:

-Yā Mi Señor, a tí/es la loaçiyón, i l-agà radeçimiy ento,/ Tú es mi Señor, el de la nobleza, i -`el/de la onrra, - el/de la

f. 53v Depu"es pensé/\{pensé \} kon deçender de poder//de mi Señor, i k'ridóme, i díšome:/

-Yã Muḥammad, yo kiv ero [a]debdeçer ${ }^{9}$ sobre tú, $\mathbf{i}$

5 sobre tu alumma en ake/sta noche, un debdo, kiven kun*/ p'lirã kon -él, pu" es a él es el -aljanna,/i ki' en defalleçerá, $\mathrm{pu}^{\mathrm{w}}$ es/ konta $^{\mathrm{a} r a \mathrm{r}^{\mathrm{y}} \mathrm{o}}$ será sobre mí, si kerré,/perdonarlé, i si kerré parçiré d-él./

I diše él:

$10-$ Yã mi Señor, i mi Mayor," /el oir es a tú, i ỳ a mí ell -obedeçer.//

i. $54 \mathrm{r}$ Adebdeçi 'ó sobre $\mathrm{mi}$ i sobre mi/alumma çinku" enta a'șşalaes de/díy a i de noche.

Depu" es deballé del poder de mi Señor,/i -y èl hazí' a salu-

5 taçiy ón*/sobre mí i sus almalakes, I de/çendí del poder de $\mathrm{mi}$ Señor alegre/i gozoso, porke apíy a fecho donaçiy ón/a mí de la ivantalla, i de la onrra.

10 Depu" es/tomóme un almalak de los almalakes, $/ \mathrm{i}$ fu" ese kon mi enta Cidratu almuntahā,/i to robé a Jibrīl, calayhi f. 54v iıççalām, // \{vi\} parado en su lugar, ke no s-abí' a mudado, $/ \mathrm{i}$-v en su mano la derecha, un mançebo muy her/moso de kara, kon muy ga ran parba,/i dišo:

-Yā Jibrïl, ¿kì én es esti?*/

Dišo a mí:

- Yã Muhammad, akesti es tu ermano/Múçã, ' alayhi i'ççalam, adélāntate, yä/Muhammad, i da a' ççalam sobr-él.

Dišo.

I -' ade/lantéme, i doné a'ççalām sobr-él, i tornó/sobre mí el -açaläm. i dišo:

$10-\mathrm{Bi}^{y}$ en seas*/penido, yā Muhammad, kon el -ermano f. $55 \mathrm{r} \quad \mathrm{pu}^{\text {" eno (sie) } / \mathrm{y} \text {-annabi onrrado enta Al. lah. Alégrate, } / / \text { yã }}$ Muhamad, ke tú es el más onrrado de las jentes ent-ada Al.lah, ¿komo/á seyido tu venida enta Al.lah? Dišo.

-Muy/biy en rrecebido - diso - ke tú eres*/enta Al.ah de ga ran p' reç; enbero, ade/bdeçiyó sober tú kosa alguna? Dišo: /

-Adebdeçiץ ó sobre mí i sobre mi alumma/çink" enta a'ș̣alaes ent" re el díy a/i la noche. Dišo:

9 El alif inicial va sin vocal, suponemos que por descuido. 
10 -Yä Muhammad, tórrnate a tu Señor,*/i demándale ke te aliyaneçka a -ellos,/ke tu alumma no bastarán sobre ellos.//

f. $55 \mathrm{v}$ I dišo:

-Yã Müçā, i kiy én osará kome/ter a los espantos akellos ke é kometido, i los milagros/akellos ke é visto?

5 Veos kon*/un ki ridante de par de mi Señor, ke dišo:/

-Yä Muhammad, demanda de mí lo ke kerrás,/ke yo soy açerca de tú, i no çeso/de demandarle, i ${ }^{-\gamma}$ alibyaneçerlle/ de mí.

10 I Müça ke mi tornase d'- ikí -y $\mathrm{a}^{*} /$ ke me hizo parar mi Señor. I me dišo mi / Señor:

f. $56 \mathrm{r}$

-Alégrate, ke ya é pu" esto//a tú i-y a ellos walardón de los cinku"ven/ta a salaes todos, por onrra de tú./yā $\mathrm{Mu}$ -

5 hammad, ke ki en hará, de tu alumma,/una alhaçana, es$\mathrm{k}^{\mathrm{e}}$ rebirle a él*/di eç $\mathrm{a}^{1}$ ḥaçanas, $\mathrm{i} \mathrm{ki}^{\mathrm{y}}$ en hará un/pekado, no le daré pena, más de por un pekado, todo akello por onrra de tú, yä Muhammad.

10 Depuw es ke Jibril, ' alayhi a ç̧alam, me tomó de mis manos, "pu" es no pasé kosa de los çiyelos, / ke no fuces sobrello eskirito://

f. 56v "lā Ilāha il-la l.lah, Muhammad r.raçūlu Al.lah"./

Depu" es tomóme Jibrîl, ' alyhi 'ççalām, de mi mano la

5 mano la derecha, $\mathrm{i}$-v ent ${ }^{\circ}$ ró $\mathrm{kon} \mathrm{mi} / \mathrm{al}$-aljanna, $\mathrm{i}$ vi sus $a^{1}$ qașares, ${ }^{*} / \mathrm{i}$ sus árboles sobre las orillas/del -aljanna, i vi el árbol de Tūbā,/akel ke $\mathrm{p}^{\mathrm{a}}$ lanató Al.lah kon la mano/de

10 la potestad, ke konpe rende las/alhaçanas todas sobre los arre*/os, i joyas; sobre kada foja d-ellas,/un almalak. i

f. $57 \mathrm{r}$ $\mathrm{e}^{10} \mathrm{su}$ mano, una seña//de $\mathrm{k}^{\mathrm{a}}$ laredad, i -` él deżi`a: "lā Ilāha il.la Al.lah,/Muhammadda r.raçūlu Al.lah". I diše:

-Yā Jibrīl,/ ¿para kiyén es esto? Dísome:/

-Para tú, yä Muhammad, i para los de tu alumma," / faga salutaçiyón Al.lah sobre tú".

Depu" es decendí por la eskalera,/i -y akesto a medi ${ }^{y} a$ noche.//

10 Falta el nun de la preposición én. Puede tratarse de un olvido o de escritura fonética. 
Excepto el ms. 1163, sigo en la transcripción los principios expuestos en mi edición ${ }^{9}$. Es decir que la transcripción en letras latinas de textos escritos con caracteres árabes debe ser tan exacta, que a base de ella se pueda reconstruir el texto en su forma original. Para no cargar demasiado el texto para un lector poco acostumbrado, he simplificado la grafía, pero he señalado las formas originales al pie de página. Así, el lector encuentra en el texto mismo podrän $(17,178$ y 16$)$ y debajo pongo bodran. Otro principio resulta del hecho que el escriba morisco emplea el alfabeto árabe para dos lenguas, el español y el árabe, porque dentro del texto español se encuentran citas árabes. Ahora, como para dos sistemas fonológicos el morisco emplea un único sistema gráfico, yo también empleo un solo sistema de transcripción para las dos lenguas. Si el morisco escribe $a^{l} s s a^{l} \bar{a} m u(17,176 \mathrm{v} 15)$ con "sin", mi principio me obliga a transcribir tersera $(17,176 \mathrm{v} 16)$, porque el morisco lo escribe también con "sin". Confieso que no encuentro ideal esa manera de transcribir palabras españolas.

La "šin" que el morisco usa en palabras árabes, por ejemplo en yušrikūna $(17,176 \mathrm{v} 15)$, la emplea también en palabras españolas. Por eso transcribo šemeğanteš. En este caso dudo menos, porque se trata por cierto de un uso lingüístico corriente entre los moriscos. Donde el español cristiano decía $s$, los moriscos solían articular $\check{s}^{10}$.

En el caso de la $\vec{a}$ larga hago una distinción. La transcribo $e$ en palabras españolas porque es cierto que con la $\bar{a}$ larga el morisco haya querido expresar la e española, por ejemplo en adelante $(1717 \mathrm{v}$ 1). Pero mantengo la $\bar{a}$ en las palabras árabes, porque el sistema fonológico del árabe no conoce la $e$. Así transcribo y $\vec{a}$ Muhammad $(17,176 \mathrm{v} 6)$.

Mi principio no es completamente satisfactorio sobre todo en cuanto a la "sin" ( $a^{l} s s a^{l} \bar{a} m u$ vs. tersera) y en cuanto a la "a" larga (adelante vs. $y \vec{a}$ ). Se impone la pregunta: ¿Cómo habría escrito un morisco, si hubiera empleado letras latinas? La respuesta a esta pregunta se encuentra en los pocos manuscritos escritos en letras latinas. Uno de ellos es el ms. 60. Este texto podría servirnos de modelo para la transcripción de los mss. en caracteres árabes.

Dos objeciones me impiden todavía aceptar el sistema gráfico del ms. 60 inmediatamente y sin reservas. En primer lugar el ms. me parece bastante moderno. El catálogo de Ribera Asín ${ }^{11}$ lo fecha a "mediados del siglo XVII". Hay aquí un enigma porque los moriscos fueron expulsados en 1609. En segundo lugar, el sistema gráfico del ms. no es coherente.

${ }^{9}$ Aljamiadotexte, pp. 25 ss.

${ }^{10}$ Cf. Aljamiadotexte, pp. 43 ss.

11 J. RIBERA y M. ASIN, Manuscritos árabes y aljamiados de la Biblioteca de la Junta, Madrid 1912, p. 213. 
En principio, este ms. tiene para la oclusiva velar las letras $c$ resp. $q$ (con 144v 9; qe 144v 14). Para la africada dental el ms. tiene $q$ resp. c (çiento 144v 7; terçera 144v 14; meçquitas 145r 4; berguença 147r 9). Pero las desviaciones son mucho más numerosas que los casos normales y se tiene más bien la impresión de un gran caos. Así, el morisco escribe casi siempre formas como çon, çosas, çomençé, çoraçón, peçado, çinçuenta, etc. (Las palabras se encuentran a pie de página).

La $s$ en partes españolas se escribe en este ms. como $s$, por ejemplo sus (144v 3) sosegadas (144v 9). Cuando el morisco escribe $x$, se trata de la fricativa prepalatal, por ejemplo dixo (144 v 18).

Del lado árabe el morisco no ve ningún inconveniente en expresar el sin y el şäd por $\varsigma$, por ejemplo açalemu (144v 18), Idriç (145v 7), çala (145v 21), ̧̧ubhana (144v 22). Pero esta grafía no es empleada estrictamente. Lo demuestra la forma acalaes ( $147 \mathrm{r} 4$ ) en vez de açalaes

El morisco sabe que hay un medio para distinguir la africada dental de la oclusiva velar, pero no sabe hacer un uso coherente de estas letras. Se equivoca muchas veces. Un editor moderno podría aceptar la intención del escriba morisco y realizarla de una manera sistemática. Yo mismo no he podido decidirme aún a transcribir esos textos según el modelo del morisco. Sólo he planteado el problema. Pero es muy probable que la fórmula para las ediciones de textos aljamiados será "TRANSCRIBIR LOS TEXTOS COMO LOS HABRİA ESCRITO UN MORISCO EVITANDO SUS INCOHERENCIAS".

Las ediciones de Galmés de Fuentes están ya muy cerca de esta concepción $^{12}$. El escriba del ms. 60 escribe siempre una $e$ donde el árabe pone una a larga. Así leemos en su texto: ye (144v 10) para $y \bar{a}$, baraquetuhu (144v 19) para barakätuhu y açalemu (144v 20) para as-salämu. Un editor moderno también tendría que tomar en cuenta este hecho.

¿Qué relaciones existen entre los 5 textos? Podemos reconocer dos grupos que se distinguen por su disposición, su contenido y su lenguaje. Aquí no puedo tratar el asunto exhaustivamente ${ }^{13}$. Basten las observaciones siguientes: tenemos por un lado los textos de 9 y de 5053 y por otro los de 17,60 y 1163 . He aquí unos rasgos diferenciales: en el centro del interés de nuestro pasaje están en todos los textos 1) el interrogatorio de parte de Allah y 2) el regateo con Allah sobre los deberes religiosos, pero en los dos grupos la disposición es distinta y cada grupo contiene elementos que el otro no tiene.

$\mathrm{Al}$ principio de todos los textos se lee que Mahoma se encuentra cerca de o se acerca a Allah. Después vienen las diferenciaciones. En

12 A. Galmés de Fuentes, Historia de los amores de París y Viana, Madrid 1970; y El libro de las batallas, 2 ts., Madrid, 1975.

13 Me propongo editar juntos los mss. que tratan el tema de la ascensión de Mahoma. 
el primer grupo $(9,5053)$ se aprende que Aliah le impone a Mahoma su mano y hace entrar en él el saber. En el otro grupo (17, 60 y l163) Allah interpela a Mahoma tres veces, el cual tiembla de miedo al principio para sosegarse después. Sigue un intercambio de salutaciones y de fórmulas piadosas entre Mahoma y Allah. Después los dos grupos tienen un tema común: un interrogatorio por parte de Allah. De él hablaré más tarde. Por el momento tenemos que seguir la sucesión de las ideas. La última expresión en el interrogatorio es el așsala (es decir "la oración") de la noche. Por eso es natural que en el primer grupo $(9,5053)$ Allah encargue a Mahoma de llevar la oración a su comunidad ("lleva ell-așsala a tu alumma").

Esa orden conduce orgánicamente a una discusión sobre el número de las oraciones cotidianas. Es éste el famoso regateo entre Allah y Mahoma que nos recuerda la discusión entre Dios y Abraham acerca de los justos en Sodoma en Génesis 18, 20 ss. Mahoma consigue rebajar el número de las oraciones cotidianas de 50 a 5 . Algo análogo sucede con los días del ayuno que Mahoma logra rebajar de 60 a 30. Después Mahoma se despide de Allah y vuelve a encontrar a Gabriel con Riḍ̂ãn, el portero del paraíso.

En todo el relato hay una sucesión orgánica de ideas. El último tema del interrogatorio es la oración. De ahí resulta la orden de llevar la oración a la comunidad musulmana. Y esto nos conduce al regateo análogo acerca de los días del ayuno.

No encontramos la misma sucesión armoniosa de las ideas en el segundo grupo $(7,60,1163)$. Al final del interrogatorio falta la orden de llevar la oración a la comunidad islámica. Además hay varias intercalaciones entre el interrogatorio y el regateo acerca de las oraciones. Esas intercalaciones parecen a veces bastante confusas. Entre ellas hay esa alusión a la espada de la guerra $(17,177 \mathrm{v} 2 s s .60$, 145v, l ss.; 50v 8 ss.). Después Mahoma reprocha a Allah haberse mostrado generoso con otros personajes, con Abraham, Idris, David y Salomón. Allah le tranquiliza y le enumera todos los regalos que ha recibido y que recibirá aún y que son mucho más importantes que los bienes acordados a los demás. $(17,177 \mathrm{v}, 6$ ss.; $60,145 \mathrm{v}, 6$ ss.; $1163,51 r, 5$ ss.). Y sólo ahora viene la discusión sobre el número de las oraciones en la cual Moisés interviene directamente $(17,178 \mathrm{v}, 14$ ss.; 60, 146r, 9 ss.; 1163, 53r, 10 ss.). Después Mahoma continúa su viaje. En este segundo grupo la sucesión de las ideas no es tan clara como en el primero. Se encuentra alterada por la intercalación de otros temas que me parecen posteriores. Por eso creo que el primer grupo es más antiguo. Pero esta impresión necesita ser confirmada por el examen de otros pasajes, lo que no puedo hacer en tan breve tiempo.

Mi división en dos grupos se ve justificada también por el vocabulario. Examinemos por ejemplo el interrogatorio. Cada uno 
de los dos grupos tiene su propio vocabulario. Sólo cito el comienzo de este pasaje.

Donde el primer grupo dice:

¿En ké konti ${ }^{y}$ enden loš de la korte alta? $(9,34 \mathrm{v}, 11$ ss.). resp. ¿En ké konti enen loš de la korte alta? $(5053,14 \mathrm{r}, 3$ ss.) Leemos en el segundo grupo:

¿Sabes kon ké peleytean la konpaña alta? $(17,176 \mathrm{v}, 16$ ss.). resp. ¿Sabes en qué pleitean la qonpaña alta? $(60,144 \mathrm{v}, 23$ ss.). resp. ¿Sabes en kée peleyteen la konpaña? $(1163,49 \mathrm{v}, 7)$.

La comparación de más pasajes conduce al mismo resultado.

Es pues evidente que hay dos grupos de textos. Probablemente el grupo 9/5053 es más antiguo que el de 17/60/1163. Establecer dependencias dentro de cada grupo es aún prematuro.

Hablemos ahora de un problema del contenido. En el interrogatorio Allah pregunta a Mahoma ¿"ké disputa le korte alta?" resp. "la konpaña alta". Esta expresión debe ser una traducción del coránico al-mala'u l-ac lā (Cor. 37, 8 y 38, 69) "el Consejo Supremo" (de los Ángeles). El tema de esta corte en nuestros textos es de ganar grados altos y de faser derremisión. Después se habla de la alumma, de la comunidad islámica (en $9,34 \mathrm{v}, 15$ y $3505,14 \mathrm{r}, 7$ ). ¿Se refieren "los grados altos" y la "derremisión" a los ángeles o a la comunidad islámica? ¿Es que los ángeles hablan de la posibilidad de derremisión de los musulmanes? El pasaje no es claro. Tampoco se hace más claro cuando lo comparamos con un hadit, una tradición, que nos ofrece Ibn Hanbal ${ }^{14}$.

En este hadit se le pregunta a Mahoma: "Sabes sobre qué se disputa en el Consejo Supremo?" Y Mahoma contesta "Sí, se disputa sobre las satisfacciones y los grados". Y el hadit continúa "¿Y qué son las satisfacciones y los grados?" Y la respuesta es: "Estar en las - mezquitas y dirigirse a pie a las oraciones comunes y ejecutar exactamente la ablución en situaciones adversas". Se ve que este pasaje de un hadit tiene mucho en común con los textos de los dos grupos. Pero el sentido queda oscuro.

También la parte final del hadit coincide con nuestros textos. Leemos en Ibn Hanbal: "Los grados consisten en la repartición de víveres, en la difusión del saludo y en la oración nocturna mientras la gente está durmiendo".

En este interrogatorio se trata de la kaffāra, lo que los textos llaman la "satisfacción", resp. la "derremisión". Pero ¿por qué ocupa ese término en lugar tan alto? Además: la satisfacción afecta a

It Ibn Hanbal, Musnad, El Cairo, 1949, nưm. 3484. También en Tirmidí, Sạịỉ. Bưlậq 1292 h. t. 2 , p. 214. 
los seres humanos. ¿Por qué los ángeles disputan sobre ella? Y, ¿qué son los "grados"? Los pasajes de nuestro texto y el hadit no bastan para resolver el problema. Tendré que continuar la bủsqueda por el lado islamológico. No se puede estudiar textos aljamiados sin recurrir a la lengua árabe y a la islamología.

También los problemas lingūísticos son varios. Sabemos ya que los textos aljamiados se caracterizan por sus arcaísmos, sus rasgos dialectales aragoneses y sus arabismos ${ }^{15}$. Esto se nota también en el vocabulario.

Para los arcaimos y los aragonismos cito un ejemplo de cada uno. Una expresión arcaica es la palabra ende en la frase: "Ya t-end-é quitado d-ellos dieç" (60, 146v 21). Un aragonismo es la preposición enta que se encuentra varias veces en nuestros textos. Baste un ejemplo: "I fü ${ }^{w}$ ese kon mí enta Çidratu almuntahá" (1163, $54 r, 10)$.

Me detendré un poco más en los arabismos. He aquí la lista de las palabras árabes de nuestros textos. Las cito en la forma que ofrecen los textos, pero no indico todas las variantes:

albakara: bendición

al' arš, alargi: trono

alchama: compañía

aldduni y a: mundo

alğanna: paraíso

alhasana: obra buena

almalak: ángel

almasğid: mezquita

alqașar: castillo

alumma: comunidad religiosa

aramadan: mes de ayuno

$\left.\begin{array}{l}\operatorname{arrak}^{c} \mathrm{a} \\ \operatorname{arrak}^{c} \text { amiento }\end{array}\right\}$ inclinación

arrak $^{c}$ ado inclinado

\author{
$\left.\begin{array}{l}\text { asağda } \\ \text { asoğdamiy to }\end{array}\right\}$ postración \\ açachedado: postrado \\ annabi: profeta \\ așșafe: fila \\ așșala: oración \\ assalám, açalen: saludo \\ $a^{1}$ taçbihar: alabar a Dios \\ a țtahur: purificación \\ alwaḍū, alguador: ablución ritual \\ yá, ye: oh! (partícula vocativa) \\ adalafe (?)
}

Dos nombres necesitan una explicación: Sidratu Elmuntahá $=\mathrm{el}$ azofaifo del límite, árbol al extremo del séptimo cielo; Țủbá=el árbol de la felicidad.

Como en otros textos aljamiados notamos también aquí que palabras árabes se integran en el sistema morfológico español. Lo demuestran los participios $a r r a k^{c}$ ado y açachedado y los sustantivos arrak'ami $i^{y}$ ento y asuğdami $i^{\prime} e n t o$. De la misma categoría es la palabra alguador cuya identificación sería algo difícil si no conociéramos el texto árabe correspondiente, donde se lee al-wuḍu ${ }^{16}$. Hasta

\footnotetext{
15 A. Galmés de fuentes, París y Viana, p. 217.

${ }^{16}$ Ibn Hanbal, Musnad 3484.
} 
ahora no he podido identificar la palabra adalafe. Una edición completa debe ofrecer también la traducción de las frases y fórmulas árabes del texto.

También en palabras puramente españolas hay problemas. ¿Cómo se explica la palabra almbiy amiy ento? $(9,35 \mathrm{v}, 1)$. No cabe duda que la palabra se relaciona con alivio. En 1163 el pasaje correspondiente ofrece la forma verbal alibyaneçka (1163, 55r, 10). La $n$ superflua en alinbiy amiy ento se explica quizá por la interferencia de limpio, que en aljamiado es linpiy o.

Como ejemplo de la abundancia de arabismos sintácticos cito sólo una frase en la que encontramos una oración nominal y una oración de estado. Una de las condiciones para obtener "los grados" es "el-așala en la noğe i las gentes durmiy endo" (9, 35r, 1 ss.), es decir "la oración en la noche mientras la gente está durmiendo". El texto en 5053 es idéntico. 1) "Las gentes durmiendo" es una oración nominal que sirve 2) de oración de estado, en la frase completa. Que se trate de una interferencia del árabe es fácil de demostrar. En el texto árabe correspondiente (Ibn Hanbal, Musnad No. 3484) leemos: wa-ș-șalátu bi-l-layli wa-n-näs niyām, que es literalmente "la oración y la gente durmiendo".

Termino aquí la presentación de 5 variantes de una pequeña parte del "puyamiento" de Mahoma a los cielos. He mostrado algunos problemas y he podido proponer algunas soluciones. Sé que este pasaje contiene aún más dificultades. Sé también que para resolverlas se debe tomar en cuenta todo el texto. Y sobre todo: nada puede ser escrito definitivamente sin recurrir a los originales árabes y sin informarse en la islamología. En este sentido me propongo continuar mis trabajos.

REINHOLD KONTZI

Tübingen. 